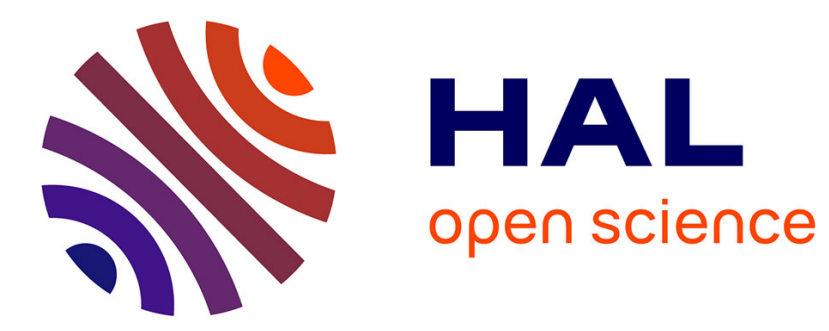

\title{
Gas-induced variation in the dielectric properties of carbon nanotube bundles for selective sensing
}

F. Picaud, R. Langlet, Madjid Arab, M. Devel, C. Girardet, S. Natarajan, S. Chopra, A. Rao

\section{- To cite this version:}

F. Picaud, R. Langlet, Madjid Arab, M. Devel, C. Girardet, et al.. Gas-induced variation in the dielectric properties of carbon nanotube bundles for selective sensing. Journal of Applied Physics, 2005, 97 (11), pp.114316. 10.1063/1.1906289 . hal-01872984

\section{HAL Id: hal-01872984 \\ https://hal.science/hal-01872984}

Submitted on 26 May 2021

HAL is a multi-disciplinary open access archive for the deposit and dissemination of scientific research documents, whether they are published or not. The documents may come from teaching and research institutions in France or abroad, or from public or private research centers.
L'archive ouverte pluridisciplinaire HAL, est destinée au dépôt et à la diffusion de documents scientifiques de niveau recherche, publiés ou non, émanant des établissements d'enseignement et de recherche français ou étrangers, des laboratoires publics ou privés. 


\title{
Gas-induced variation in the dielectric properties of carbon nanotube bundles for selective sensing
}

\author{
F. Picaud, R. Langlet, M. Arab, M. Devel, and C. Girardet \\ Laboratoire de Physique Moléculaire, Unité Mixte de Recherche (UMR) 6624, Faculté des Sciences, \\ La Bouloie, Université de Franche-Comté F25030 Besançon Cedex, France
}

S. Natarajan, S. Chopra, and A. M. Rao ${ }^{\text {a) }}$

Department of Physics and Astronomy, Clemson University, Clemson, South Carolina 29634

(Received 3 December 2004; accepted 21 March 2005; published online 27 May 2005)

\begin{abstract}
There is an increasing demand for robust, miniaturized sensors with ppm or parts per $10^{9}(\mathrm{ppb})$ sensing capability, and high selectivity to different chemical or biological species. Here we show that trace amounts (ppb) of gases or organic solvent vapors can be detected with high selectivity and sensitivity using single-walled carbon nanotube bundles in a resonator configuration. The enhanced sensing properties result from a change in the effective dielectric properties of the resonator when exposed to different gas environments. A theoretical model is described which computes resonant frequency shifts that are in remarkable agreement with corresponding experimental shifts exhibited by the resonator when exposed to different gas molecules. This work demonstrates a gas-sensing platform with superior sensitivity and selectivity for gas detection, and presents advantages in terms of portability and recovery time. In particular, the sensing platform does not require functionalized carbon nanotubes to enhance specificity, or wire connection to the nanotubes making it attractive for remote sensor technology. (C) 2005 American Institute of Physics. [DOI: 10.1063/1.1906289]
\end{abstract}

\section{INTRODUCTION}

The exceptional physiochemical properties of carbon nanotubes, especially their high surface area, chemical inertness, and their geometry-dependent electrical properties (metallic or semiconducting character), ${ }^{1}$ make them attractive for several applications in the field of miniature gas sensors. ${ }^{2-5}$ Experimental $^{6,7}$ and theoretical ${ }^{8,9}$ reports on the adsorption sites and binding energies of various simple molecules suggest appreciable adsorption energies (from 100 to a few hundreds of $\mathrm{meV}$ ) on single-wall nanotube (SWNT) bundles when compared to the thermal energy $(25 \mathrm{meV})$ at room temperature. Sensors based on SWNTs exhibit high sensitivity, short response, and recovery times compared to traditional sensors. Detectable changes in the electrical resistance measured at room temperature have been reported ${ }^{5,10}$ when ppm amounts of $\mathrm{O}_{2}, \mathrm{NO}_{2}$, or $\mathrm{NH}_{3}$ molecules are adsorbed on SWNT films. Other electrical properties of nanotubes, such as thermoelectric power, ${ }^{11}$ electrical breakdown voltage, ${ }^{12}$ and dielectric properties, ${ }^{13}$ have also been suggested as useful indicators for sensing since these properties can change significantly upon exposure to specific gases. While all of the above possess respectable sensing capabilities, they have severe limitations for realistic applications. For example, different types of gases present in a mixture of gases cannot be readily discriminated, and most sensors require wire connections that preclude their use in remote sensor technology.

In this paper, we describe an innovative sensing platform in which resonant frequency shifts induced by adsorbed molecular species on SWNT bundles are highly specific to the nature of the adsorbed species. We further correlate experi-

\footnotetext{
a) Author to whom correspondence should be addressed; electronic mail: arao@clemson.edu
}

mentally observed shifts with computed changes in the linear electronic susceptibility of the resonator due to molecular adsorption. This type of sensing has significant advantages over other sensors discussed in the literature and provides research directions for chemical or biological sensing applications.

\section{EXPERIMENTAL DETAILS}

A schematic of our circular disk resonator and the gassensing setup is described in Fig. 1. The resonant frequency $f_{0}$ of the resonator is related to its dielectric constant $\varepsilon_{r 0}$ as $f_{0}$ $\alpha 1 / \sqrt{\varepsilon_{r 0}} .{ }^{14}$ In a perfectly matched resonator, the electromagnetic field couples to the circular disk solely through the dielectric (Duroid board) without leaking into the surrounding environment. In our design, this condition is relaxed so that part of the electromagnetic-field lines couple to the circular disk by passing through the environment above the disk. Hence, different gases when introduced into the testing chamber adsorb on the nanotubes present on the circular disk and alter the effective dielectric constant $\varepsilon_{r}$ of the resonator in proportion to the dielectric properties of the gas species. The presence of a particular gas is evidenced as a shift $\Delta f$ in the resonant frequency due to a change in $\varepsilon_{r}$. Further, since our resonator operates in the microwave regime, a small change in $\varepsilon_{r}$ triggered by the presence of parts per $10^{9}(\mathrm{ppb})$ amounts of any gas leads to a discernable shift $\Delta f$ $=f_{0}\left(\sqrt{\varepsilon_{r 0} / \varepsilon_{r}}-1\right)$. This uniqueness in its design facilitates selective room-temperature detection of a range of gases or organic solvent vapors. First, the SWNT bundles are degassed by evacuating the testing chamber to $10^{-5}$ torr and simultaneously heating the chamber to $\sim 130{ }^{\circ} \mathrm{C}$. Next, the testing chamber is cooled to room temperature while it is maintained under high vacuum and the frequency is recorded 


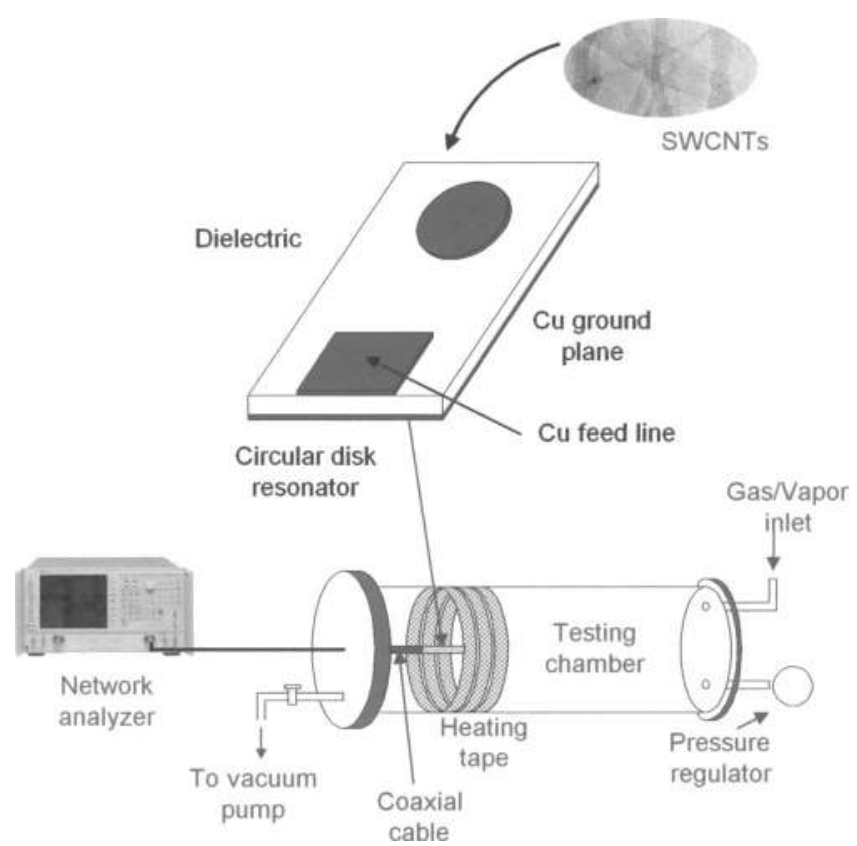

FIG. 1. Schematic of the circular disk resonator (top) and the gas-sensing setup (bottom). As-prepared, electric-arc-prepared SWNT bundles are physically coated on the conducting $\mathrm{Cu}$ disk using a conductive epoxy (Circuit Works CW2400) and the resonator is placed inside the vacuum tight glass chamber. Electromagnetic energy is coupled into the resonator through a rectangular microstrip feed line. Varying concentrations of gases when introduced into the chamber lead to shifts in the resonant frequency which is recorded by the network analyzer.

by the network analyzer before and after exposing the resonator to a known amount of gas introduced into the chamber.

\section{RESULTS AND DISCUSSION}

As seen in Fig. 2, the shift $\Delta f$ is relatively large for vapors of acetone compared to the corresponding shift for bromopropane. Another unique advantage of this sensor is its ability to detect vapors over a wide concentration range spanning from $100 \mathrm{ppb}$ to $1500 \mathrm{ppm}$ without saturating the sensor [Fig. 2(c)]. This observation is attributed to the high specific surface area of SWNT bundles relative to other types of carbon nanotubes, such as multiwalled carbon nanotubes. ${ }^{15}$ The ability to selectively detect ppb levels of gases coupled with the broad concentration range of detection makes the present sensor configuration superior to contemporary nanotube-based sensors. Finally, it should be noted that the sensing mechanism stems from a change in $\Delta f$; therefore the purity or the amount of residual catalyst has a minimal effect on the sensing properties of our sensor.

The shifts $\Delta f$ induced by the presence of $\sim 1500 \mathrm{ppm}$ of different vapors are summarized in Fig. 3. All measurements were carried out under similar conditions using the same resonator for each of the vapors. These shifts scale proportionally as a function of the dielectric constant $\varepsilon$ of the vapors. As a result, organic vapors with higher dielectric constants lead to a greater shift compared to the gases such as nitrogen or oxygen, implying that our sensor also provides high selectivity for gas detection. The $\Delta f$ for the resonator when exposed to a mixture of organic solvent vapors is also shown in Fig. 3. Again, $\Delta f$ for the mixture can be understood (a) Acetone

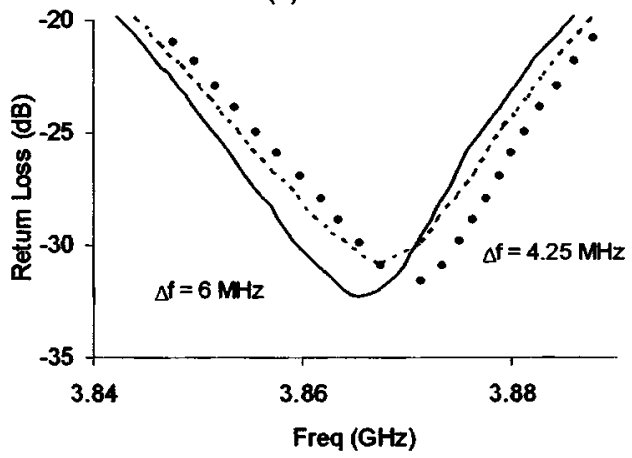

(b) Bromopropane

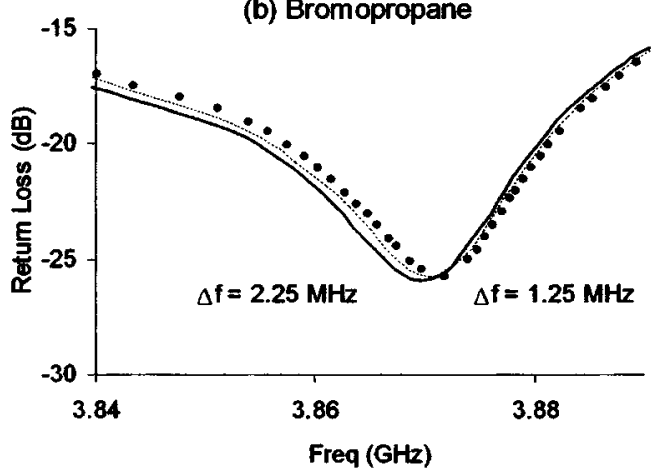

(c) Response to Acetone vapors

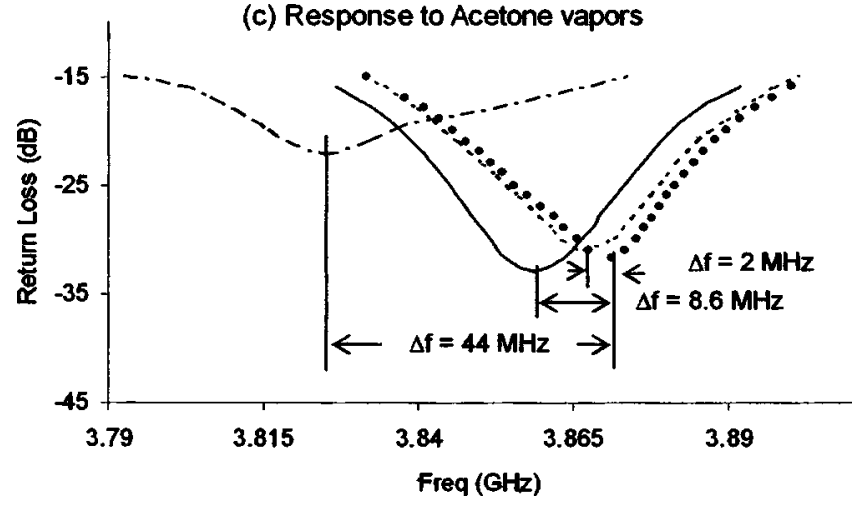

FIG. 2. (a) Response of the resonator sensor to acetone $\left(\mathrm{CH}_{3} \mathrm{COCH}_{3}\right)$ vapors. The resonant frequency shifts are measured relative to vacuum (trace defined by black filled circles) and are shifted by 4.25 (dashed line trace) and $6 \mathrm{MHz}$ (solid line trace) when exposed to 350 and $950 \mathrm{ppb}$ of acetone, respectively. (b) Response to bromopropane $\left(\mathrm{CH}_{3} \mathrm{CH}_{2} \mathrm{CH}_{2} \mathrm{Br}\right)$ vapors: vacuum (trace defined by black filled circles), 150 (dashed line trace) and $875 \mathrm{ppb}$ (solid line trace). (c) Response to acetone vapors ranging from ppb to ppm levels: vacuum (trace defined by black filled circles), $800 \mathrm{ppb}$ (dashed line trace), $15 \mathrm{ppm}$ (solid trace), and $1300 \mathrm{ppm}$ (dashed dotted trace).

in terms of $\varepsilon$ for each component present in the gas mixture. For mixtures containing more than two components, a detailed modeling for $\Delta f$ in terms of $\varepsilon$ is needed to compare with the experimentally observed values of $\Delta f$.

In addition to $\Delta f$, another evident feature in Fig. 2(c) is the decrease in the $Q$ factor of the resonator upon exposure to different concentrations of acetone. Thus, the $Q$ factor can serve as an independent variable to discriminate the presence of specific gases. Finally, it should be mentioned that the resonator can be remotely operated with a conventional transceiver system making it a remote sensing system. ${ }^{16}$

As a first step towards a realistic interpretation of the experimental results described above, we determine the po- 


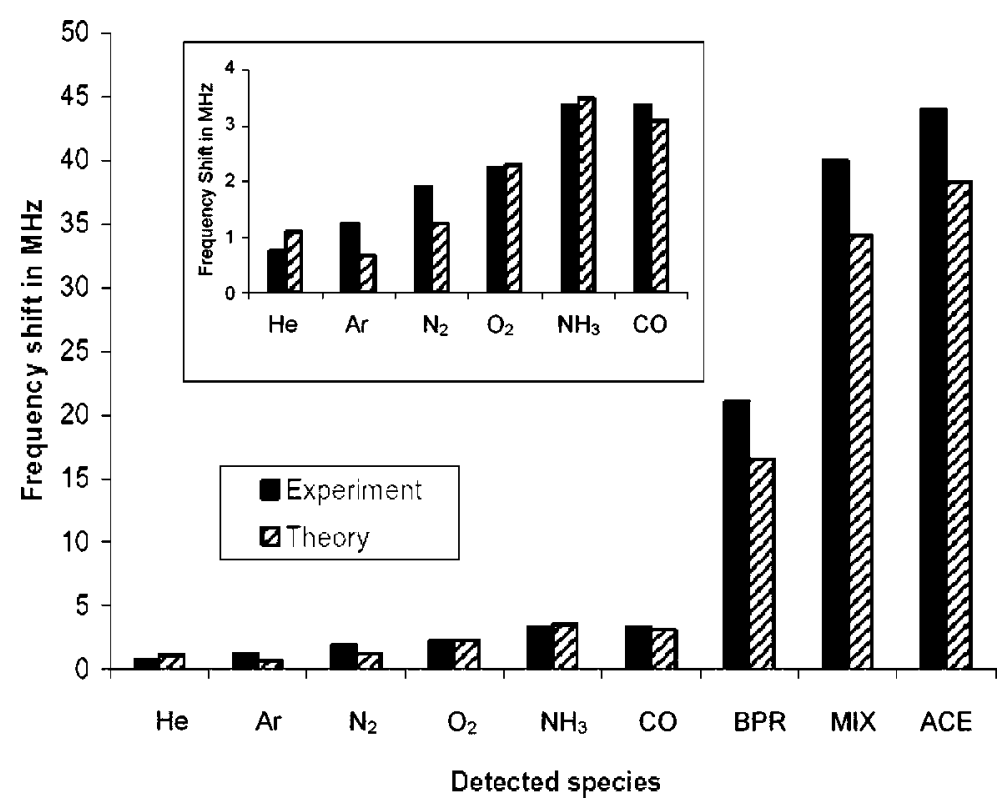

FIG. 3. Summary of experimental (solid) and theoretical (hatched) shifts $\Delta f$ when exposed to different gases and organic solvent vapors (BPR=bromopropane, MIX $=25 \%$ bromopropane $+75 \%$ acetone (v/v), and $\mathrm{ACE}=$ acetone). The inset is an enlargement of the data for the gases, only. The calculated coverages are, respectively, $5 \times 10^{-3}$ for $\mathrm{He}, \mathrm{O}_{2}, \mathrm{CO}, \mathrm{NH}_{3}$, and $\mathrm{BPR}$, $1.25 \times 10^{-3}$ for $\mathrm{Ar}, 2.5 \times 10^{-3}$ for $\mathrm{N}_{2}$, and $3 \times 10^{-2}$ for acetone, as determined by the Langmuir formula.

larizability variations of a perfect, single $(17,0)$ SWNT of length $L \sim 30 \AA$, in the presence of an external electric field $\mathbf{E}_{\mathbf{0}}$ (which can be identified to the field lines that couple the nanotube to the resonator) when gas molecules are physisorbed on its outer surface. The choice of the tube radius is consistent with most of the experimental distribution peak presenting a maximum around $7 \AA$. The values of effective polarizabilities parallel and perpendicular to the graphene sheet $\alpha_{C \mid}=2.47 \AA^{3}$ and $\alpha_{C \perp}=0.86 \AA^{3}$ for carbon atoms ${ }^{17}$ (which remarkably fit the measured polarizabilities of graphite and fullerene molecules) and of the anisotropic molecular polarizabilities for the admolecules ${ }^{18}$ are used to evaluate the dielectric properties of the resonator. The length of the nanotube and the molecular coverage $\theta$ can be varied to estimate the sensitivity of the system. The field $\mathbf{E}_{\mathbf{0}}$ is chosen weak enough to probe the dielectric response of the system within the linear-response approach, without influencing the adsorption geometry of the molecules.

The local electric field $\mathbf{E}_{\mathbf{0}}\left(\mathbf{r}_{\mathbf{i}}\right)$ at a position $\mathbf{r}_{\mathbf{i}}$ (index $i$ characterizes a carbon atom or an adsorbed molecule) is written as

$$
\begin{aligned}
\mathbf{E}_{\mathbf{l}}\left(\mathbf{r}_{\mathbf{i}}\right)= & \mathbf{E}_{\mathbf{0}}+\sum_{j \neq i}^{N_{d}}\left[\overleftrightarrow{T_{R}^{2}}\left(\mathbf{r}_{\mathbf{i}}, \mathbf{r}_{\mathbf{j}}\right) \boldsymbol{\mu}\left(\mathbf{r}_{\mathbf{j}}\right)-\frac{1}{3} \overleftrightarrow{T_{R}^{3}}\left(\mathbf{r}_{\mathbf{i}}, \mathbf{r}_{\mathbf{j}}\right) \overleftrightarrow{Q}\left(\mathbf{r}_{\mathbf{j}}\right)\right] \\
& +\sum_{j \neq i}^{N+N_{d}} \overleftrightarrow{T}_{R}^{2}\left(\mathbf{r}_{\mathbf{i}}, \mathbf{r}_{\mathbf{j}}\right) \overleftrightarrow{\alpha}\left(\mathbf{r}_{\mathbf{j}}\right) \mathbf{E}_{\mathbf{l}}\left(\mathbf{r}_{\mathbf{j}}\right),
\end{aligned}
$$

where the second term defines the molecular field due to the permanent dipolar $\boldsymbol{\mu}\left(\mathbf{r}_{\mathbf{j}}\right)$ and quadrupolar $\mathbf{Q}\left(\mathbf{r}_{\mathbf{j}}\right)$ moments in the $j$ th adsorbed molecule (with $\overleftrightarrow{T}_{R}^{2}$ and $\overleftrightarrow{T}_{R}^{3}$ renormalized propagator tensors related to $\boldsymbol{\mu}$ and $\mathbf{Q}$ ), while the third contribution describes the polarization of the $N$ carbon atoms and $N_{d}$ adsorbed molecules by the local electric field, and ensures the self-consistency of the polarization phenomenon. This self-consistent electric equivalent of the LippmannSchwinger equation is used twice, first to calculate the interaction energy between the adsorbed molecules and the nanotube, and second to determine the electronic polarization of the resonator. The interaction energy experienced by the $i$ th molecule is written as ${ }^{9}$

$$
V_{i}=-\boldsymbol{\mu}\left(\mathbf{r}_{\mathbf{i}}\right) \cdot \mathbf{E}_{\mathbf{l}}\left(\mathbf{r}_{\mathbf{i}}\right)+\frac{1}{3} \stackrel{\leftrightarrow}{Q}\left(\mathbf{r}_{\mathbf{i}}\right) \cdot \nabla \mathbf{E}_{\mathbf{l}}\left(\mathbf{r}_{\mathbf{i}}\right)+V_{\mathrm{DR}},
$$

where the first two contributions are related to the electrostatic and induction interactions and $V_{\mathrm{DR}}$ characterizes the quantum dispersion-repulsion interaction described by a Lennard-Jones potential. The minimization of this interaction leads to the adsorption site energy $E_{A}$ and to the equilibrium geometry of the $i$ th molecule adsorbed on the tube surface. The knowledge of $E_{A}$ allows us to calculate the admolecule coverage $\theta$ using the well-known Langmuir formula, ${ }^{19}$ while information on the site and geometry is required to determine the electronic polarization $\mathbf{P}$ of the nanotube + admolecules. The polarization defined conventionally as $\mathbf{P}$ $=\sum_{i=1}^{N+N_{d}} \stackrel{\leftrightarrow}{\alpha}\left(\mathbf{r}_{\mathbf{i}}\right) \mathbf{E}_{\mathbf{l}}\left(\mathbf{r}_{\mathbf{i}}\right) / V$, where $\mathrm{V}$ is the volume of the system assimilated to an empty rod of thickness $d=3.4 \AA$, radius $R$, and length $L . \mathbf{P}$ can also be expressed in terms of the linear electronic susceptibility $\chi$ and of the macroscopic field, defined as the average over the system of the microscopic field. However, from Eq. (1), it can be shown straightforwardly that, for weak and homogeneous external field $\mathbf{E}_{\mathbf{0}}$, the macroscopic field is a linear function of $\mathbf{E}_{\mathbf{0}}$ where the slope does not depend on the permanent moment while the intersect does. As a result, the polarization is given as $\mathbf{P}$ $=\varepsilon_{0} \chi \mathbf{E}_{\text {macro }}(0)+\mathbf{P}$, where $\mathbf{E}_{\text {macro }}(0)$ is the macroscopic field calculated for all the permanent moments set to zero. This remark is essential since it shows that $\chi$ can be computed independently of the presence of the permanent moments. The change of the linear susceptibility tensor with molecular adsorption is thus proportional to the molecular polarizability of the system with a coefficient that depends on the intrinsic $\alpha$ values and on the number and geometry of the admolecules. Note, in particular, that, the orientational polarization, which is a characteristic of the permanent dipole(s) of the molecules, does not contribute to this change as long as $\mathbf{E}_{\mathbf{0}}$ does not influence their adsorption geometry. By contrast, the 
coverage is a direct function of $E_{A}$, hence of these permanent moments.

The linear dielectric susceptibility of the system formed by the SWNT plus adsorbed molecules taken in a series of species ranging from nonpolar $\left(\mathrm{He}, \mathrm{Ar}, \mathrm{N}_{2}\right.$, and $\left.\mathrm{O}_{2}\right)$, weakly polar $(\mathrm{CO})$ to strongly polar $\left(\mathrm{NH}_{3}\right.$, bromopropane, and acetone) is calculated at small coverage $\theta$ to prevent lateral interactions between admolecules. The values of the coverage will be discussed below. For the non polar or weakly polar molecules, the adsorption geometry corresponds to a flat configuration (molecular axis parallel to the tube), while the dipole moment of the dipolar adspecies points towards the radial direction of the tube. The adsorption energies $E_{A}$ at $0 \mathrm{~K}$ range between $75 \mathrm{meV}$ for $\mathrm{Ar}$ and $\mathrm{N}_{2}, 97 \mathrm{meV}$ for $\mathrm{CO}$, and $147 \mathrm{meV}$ for $\mathrm{NH}_{3}$ to nearly $380 \mathrm{meV}$ for acetone and bromopropane on the external surface of the tube.

Using these adsorption geometries for the admolecules and the calculated coverage, the ratio of the dielectric constant $\varepsilon_{r}=1+\operatorname{Tr}[\chi] / 3(\operatorname{Tr}[\chi]$ means the trace of $\chi)$ for the complete system to the same quantity $\varepsilon_{r 0}$ for the SWNT alone, and the corresponding frequency shifts are calculated and compared in Fig. 3 to the measured shifts. The value for $\theta$ was chosen to match the calculated frequency shift with the observed shift for oxygen adsorption with $f_{0}$ $=3.8893 \mathrm{MHz}$. From this value of $\theta\left(\sim 10^{-3}\right.$ monolayer $)$ and the value of the adsorption energies, we applied the Langmuir formula to determine $\theta$ for all the other species investigated in this study, which in turn determine the corresponding frequency shifts of the resonator. Since the experimental frequency shifts correspond to the same dose of gas trace for all species, a close comparison with the calculations is required to determine the theoretical shifts for the same coverage. In practice, we change the tube length to account for the effective size and adsorption energy of a single admolecule as determined by the Langmuir scheme. For instance, the tube length is enhanced by a factor of 2.73 and 3.68 for acetone and bromopropane, respectively, when compared to oxygen molecule, to refer to the same coverage value calculated from the Langmuir formula. The good agreement between the experimental and theoretical shifts depicted in Fig. 3 confirms our choice $\theta$ for oxygen adsorption and is the only adjustable parameter in our model calculations. Since the ratio of dielectric constants remains close to 1.0 , the shift is approximately proportional to the relative change of the system susceptibility. Hence this shift is a quasilinear function of the mean electronic polarizability $\alpha$ of the admolecules weighted by the molecular coverage. Such a linear dependence is used to determine the shift for a gas mixture formed by $25 \%$ bromopropane and $75 \%$ acetone compared to the self-consistent calculations performed with this mixture. The good agreement between experiments and calculations for the mixture corroborates this linear law.

The fact that calculations of the dielectric response of a single short tube can interpret the experimental results of a sensor formed with a random population of SWNT bundles demonstrates that the measured resonant frequency shifts are nearly independent of the morphology of the bundles, especially of the distribution in length ( $L$ must be sufficiently long to eliminate the tube side effects), diameter size (around a given average value of $14 \AA$ ), and orientation of the nanotubes. Such a property is valid at sufficiently low coverage, i.e., for trace amounts of gas (no molecular aggregation on the tube). Incidentally, note that the tube side effects have been studied according to two ways. Cutting the tube sharply as done in this paper or, on the contrary, considering a periodic tube does not affect significantly the polarizability (while the adsorption energy can be seriously modified) when the admolecule is adsorbed at the middle of a sufficiently long tube. For instance, a value of $30 \AA$ for the tube length is reasonable to prevent side effects for small molecules $\left(\mathrm{N}_{2}, \mathrm{CO}, \mathrm{CO}_{2}, \mathrm{HF}, \mathrm{H}_{2} \mathrm{O}, \ldots\right)$, while the length must be increased for larger molecules (acetone, bromopropane, ...). The same feature is found by considering caps at both sides instead of a sharp cut of the tube. We have checked that such a change does not influence the results on the permittivity ratio. It also assumes that no strong chemisorption occurs due to the presence of defects and the possible high reactivity of some adsorbed species. ${ }^{20}$ In addition, our model disregards the possibility for molecular adsorption at sites other than those on the external surface of the tube. Within our experimental setup, a SWNT bundle could offer other sites such as groove, interstitial, and even internal sites, for which the adsorption energy values are generally much larger. ${ }^{9}$ Surprisingly, this site distribution factor is not critical for explaining the experimental dielectric response and this is attributed to the fact that molecular polarizability and the orientation of the molecule at any site are not strongly influenced by the nature of adsorption sites, as shown in another paper. ${ }^{21}$ The fact that the calculated frequency shifts linearly depend on the polarizability of the admolecule, via the square root of the permittivity ratio $\varepsilon_{r} / \varepsilon_{r 0}$, tends to strongly reduce the collective effects both on the adsorption energy and polarizability. This explains probably why, together with the independence of the polarizability on the adsorption site, the agreement appears to be excellent for most of the considered molecular species.

\section{SUMMARY}

To conclude, our sensor based on the measurement of the differential dielectric response of SWNT bundles due to molecular adsorption displays a high selectivity and sensitivity in terms of the static electronic polarizability $\alpha$ and coverage $\theta$ of admolecules via the response to an external field. A model based on the use of macroscopic electric quantities defined at the level of a single SWNT is able to interpret quantitatively the frequency shift measurements of the resonator for adsorbed nonpolar or polar molecules on the basis of these two quantities, $\alpha$, and $\theta$.

\section{ACKNOWLEDGMENT}

A.M.R. would like to acknowledge support through NSF NIRT 0304019.

${ }^{1}$ R. Saito, G. Dresselhaus, and M. S. Dresselhaus, Physical Properties of Carbon Nanotubes (Imperial College Press, London, 1998).

${ }^{2}$ P. G. Collins, K. Bradley, M. Ishigami, and A. Zettl, Science 287, 1801 (2000); C. K. W. Adu, G. U. Sumanasekera, B. K. Pradhan, H. E. Romero, and P. C. Eklund, Chem. Phys. Lett. 337, 31 (2001). 
${ }^{3}$ S. Chopra, K. McGuire, N. Gothard, A. M. Rao, and A. Pham, Appl. Phys. Lett. 83, 2280 (2003).

${ }^{4}$ S. Chopra, A. Pham, J. Gaillard, A. Parker, and A. M. Rao, Appl. Phys. Lett. 80, 4632 (2002).

${ }^{5}$ J. Kong, N. R. Franklin, C. Zhou, M. G. Chapline, S. Peng, K. Cho, and H. Dai, Science 287, 622 (2000).

${ }^{6}$ H. Ulbricht, G. Moos, and T. Hertel, Phys. Rev. B 66, 075404 (2002).

${ }^{7}$ B. Y. Wei, M.-C. Hsu, Y.-S. Yang, S.-H. Chien, and H.-M. Lin, Mater Chem. Phys. 81, 126 (2003).

${ }^{8}$ J. J. Zhao, A. Buldom, J. Han, and J. P. Lu, Nanotechnology 13, 195 (2002).

${ }^{9}$ M. Arab et al., Phys. Rev. B (in press).

${ }^{10}$ L. Valentini, L. Lozzi, C. Cantalini, I. Armentano, J. M. Kenny, L. Ottaviano, and S. Santucci, Thin Solid Films 436, 95 (2003).

${ }^{11}$ G. U. Sumanasekera, C. K. W. Adu, S. Fang, and P. C. Eklund, Phys. Rev.
Lett. 85, 1096 (2000).

${ }^{12}$ A. Modi et al., Nature (London) 424, 171 (2003).

${ }^{13}$ O. K. Varghese, P. D. Kichambare, D. Gong, K. G. Ong, E. C. Dickey, and C. A. Grimes Sens. Actuators B 81, 32 (2001).

${ }^{14}$ R. E. Collin, Foundations for Microwave Engineering (McGraw-Hill, New York, 1992).

${ }^{15}$ M. Cinke et al., Chem. Phys. Lett. 365, 69 (2002).

${ }^{16}$ K. G. Ong and C. A. Grimes, Smart Mater. Struct. 9, 412 (2000).

${ }^{17}$ M. Devel et al., Phys. Rev. B 53, 13159 (1996).

${ }^{18} \mathrm{~J}$. O. Hirschfelder et al., Molecular Theory of Gases and Liquids (Wiley, New York, 1954).

${ }^{19}$ I. Langmuir, J. Am. Chem. Soc. 38, 2221 (1916).

${ }^{20}$ N. Chakrapani et al., J. Phys. Chem. B 107, 9308 (2003).

${ }^{21}$ R. Langlet, M. Arab, F. Picaud, M. Devel, and C. Girardet, J. Chem. Phys. 121, 9655 (2004). 